\title{
Real-time delay statistics in wireless IP networks
}

\author{
D. Huo \\ Bell Laboratories, 67 Whippany Road, Whippany, NJ 07981, USA \\ Correspondence to: D. Huo (dhuo@ieee.org)
}

\begin{abstract}
In the wireless communication, the variation of the transmission delay is called jitter and is one of the variables responsible for the degradation of the service quality. Jitter is present in every section of the transmission system. Its stochastic behavior depends on the technology implemented in the system and the service provided by the system. This paper focuses on mathematical modeling and phenomenological analysis of the jitter encountered by the realtime services in a wireless network. Using the data made available to the public by the wireless industry, we explore the stochastic characterizations of the jitter in a wireless IP networks. Within the scope of real-time service, we studied the relation between delay, jitter and the inter-packet time. Evaluation of the sample data indicates a long range dependence of the inter-packet time of the received packets in a real-time connection. The result helps understanding the transmission delay encountered by the real-time service over wireless IP networks.
\end{abstract}

\section{Introduction}

To provide IP (Internet Protocol) based packet transmission service a wireless network relies on an infrastructure that consists of multiple sections. Each of these sections introduces a time delay to the packet transmission. The temporary variation of the time delay is referred to as jitter and it depends not only on the underlying transmission technology, but also on the service the packet transmission is supposed to support. The real-time service requires a fixed transmit and receive rate and, as such, its quality is impaired by the jitter. In wireless technologies such as HSPA (High Speed Packet Data) or LTE (Long Term Evolution), jitter is primarily caused by two techniques introduced to improve the radio interface performance: the packet scheduling at the RNC (radio network controller) for UMTS or at the eNodeB for LTE, and the HARQ (hybrid automatic request-response) proto- col on the radio link. The scheduling in the air-interface enables efficient resource sharing among users, and the HARQ enhances the utilization of the radio spectrum assigned to each user. In order to study the jitter caused by the airinterface in a wireless network, we consider a mobile-tomobile connection as a chain of transmission sections. In specifics, the transmission system consists of the following sub-systems in sequence: source, radio up-link, core network, radio down-link, destination. Providing real-time services such as voice requires transmission of a continuous stream of speech frames with fixed packet rate. While the packet stream has a constant packet rate at the input of the transmission system, the received packet stream at the output of this transmission system may not have the same constant packet rate, because the time between speech frames may change as result of the delay jitter. It is thus the task of system architects to find ways, e.g. via buffer design, to cope with the jitter. Finding jitter mitigation strategies while not introducing excessive delay requires a better understanding of the jitter statistics. Here lies the motivation to the work reported in this paper. To confine our scope, we investigate jitter phenomena on the radio access network specified by 3GPP (2012). Instead of analyzing the protocols, we take a phenomenological approach by regarding each sub-system in the transmission chain as a black box and evaluate the experimental results available for the black boxes. In a project by 3GPP-RAN1 (2006), the VoIP provided by an HSPA was studied through simulations, where both up-link and downlink are implemented according to the technical specifications 3GPP-RAN1 (2011). The simulation is based on a cellular network layout of 59 sectors, using 4 channel models and 4 network loading scenarios. The detailed configuration and parameters can be found by 3GPP-SA4 (2006); ITU-R (2012). The packet delay and error event observed in the simulation can be further processed to produce sample paths of jitter. Thus, 54 sample paths can be obtained as raw material for our study. 
In Sect. 2 we will discuss the definition of the random variables delay and inter-arrival time, and characterize the packet streams of constant rate in a rather general way. Section 3 is dedicated to the characterization of the compound transmission system, consisting of concatenated sub-systems. The mining of the aforementioned data for the purpose of jitter study is performed in Sect. 4, making use of the theory developed in the previous sections. In Sect. 5 we will construct and analyze the mobile-to-mobile connection using existing sample paths, before giving a conclusion in Sect. 6.

\section{Transmission system}

A packet stream is defined by indexing each packet by a unique integer $i \in \mathbf{Z}$, where $\mathbf{Z}$ is the set of integer numbers, so that consecutive packets are packets that should have adjacent indices. While being transmitted through the transmission system, packet experience delay, which can be determined by measurement at two points: One at the input port of transmission system and the other at the output port of the transmission system. The arrival time, as well as the departure time, is a mapping of the packet index to a real number $\mathbf{Z} \mapsto \mathbf{R}$, where $\mathbf{R}$ is the set of real numbers. Note that the interpretation of the term "arrival" and "departure" depends on the context, i.e. depending on the system we observe. As far as a pair of transmitter and receiver is concerned, the arrival time at the receiver is the time when the packet departs the system of transmission medium that connects the transmitter and receiver. For a packet stream with constant data rate, the time between consecutive packets at the data source should be the same. Then,without transmission impairments the arrival time at the receiver is a monotone increasing step function with a fixed increment. Let $T(i)$ denote the time when the packet with index $i$ departs the transmitter. Then $T(i)=C+i / r$ for $i \in \mathbf{Z}$, where the real number $r>0$ is the packet rate and $C$ is the reference time. It is reasonable to assume that all packets in a packet stream are generated in order at the transmitter, i.e. $T(i)<T(j)$ for $i<j$. Let $t(i)$ be the time when the same packet arrives at the receiver. Then, $t(i)>T(i)$ for all $i \in \mathbf{Z}$. Obviously, the transmission delay of a packet $d(i)=t(i)-T(i)$ is positive for any non-trivial transmission system. Moreover it in fact a random number. For the further study, we make the assumption that the delay is a random variable defined in $(\Omega, \mathbf{B}, \mathbf{P})$, where $\Omega$ is the space of random event, $\mathbf{B}$ the Borel algebra on real numbers and $\mathbf{P}$ the probability measure. Since $t(i)=C_{1}+d(i)+i / r$ with $C_{1}$ accounting for the fixed delay of the system and the reference time at the transmitter, it is readily seen that $t(i)-E\{t(i)\}=d(i)-E\{d(i)\}$, where $E\{\}$ refers to the statistical expectation.

Another related random number is the inter-packet time. As mentioned before, with the term of inter-packet time we could be talking about the inter-arrival time or the interdeparture time, depending on the context. For both cases the
Table 1. Example of re-ordering.

\begin{tabular}{cccccccc}
\hline$i$ & 0 & 1 & 2 & 3 & 4 & 5 & $\ldots$ \\
$t(i)$ & 10 & 11 & 14 & 12 & 13 & 15 & $\ldots$ \\
$\pi \circ t(i)$ & 10 & 11 & 14 & 14 & 14 & 15 & $\ldots$ \\
\hline
\end{tabular}

inter-packet time is a mapping of index $x: \mathbf{Z} \mapsto \mathbf{R}$. In particular, the inter-arrival time at the receiver is well defined by $x(i)=t(i)-t(i-1)$ for $\lim _{i \rightarrow-\infty} t(i)=0$, and we have

Proposition 1 Between the inter-packet time and the delay there are following relations

$x(i)=d(i)-d(i-1)+\frac{1}{r}$
$d(i)=d(0)+\sum_{k=0}^{i} x(k)$

For a stable transmission system, we can assume the stationarity. Then $E\{d(i)\}=E\{d(i-1)\}$ and $E\{x(i)\}=1 / r$, meaning the transmission system does not change the average inter-packet time, which is a necessary requirement of the real time service. Assuming the packet loss is handled by the upper layer protocol, we can ignore the packet loss in our study. Normally a packet with smaller index will arrive earlier that one with a larger index. But, due to the randomness of the delay, a packet with smaller index may be received later than a packet with larger index, resulting in packet disorder in the packet stream at the receiver. The requirement of order preservation for the delivery of real-time services necessitates a posterior re-ordering operation at the receiver: It is a mapping of a set of the packet indices into itself, so that the sequential order of the packets is restored as given by the source. While the re-ordering action re-shuffles the received packets for the purpose of re-establishing the original order, it modifies the receive time, resulting in jitter.

Definition $\mathbf{1}$ Let $\mathbf{Z}$ be the set of all packet indices and $\mathbf{R}$ the set of the departure time. A re-ordering operation is a mapping $\pi: \mathbf{Z} \times \mathbf{R} \mapsto \mathbf{Z} \times \mathbf{R}$ and for $i<j$

$\pi(i, t):=(\pi \circ t)(i)=\left\{\begin{array}{l}t(j), \text { for } t(i)>t(j) \\ t(i), \text { for } t(i) \leq t(j)\end{array}\right.$

The operation $\pi$ permutes the packets with respect to the indices. Thus, for a given positive $\tau \in \mathbf{R}$,

$\pi^{-1}(\tau)=\{i \in \mathbf{N} \mid(\pi \circ t)(i)=\tau\}$,

the set of indices associated with $\tau$ after the re-ordering, is non-trivial and can have more than one elements, causing the phenomena of bursty arrival time, i.e. accumulation of packets in the receive buffer at a given time. Thus, the action of reordering changes the delay statistics. An example of disorder and re-odering operation is shown in Table 1. 
Generally speaking the re-ordering introduces delay:

Proposition 2 Let $t(i)$ be the departure time of packet $i$ and $\operatorname{Var}\{t(i)\}<\infty$. Then, for the re-ordering function $\pi: \mathbf{Z} \times$ $\mathbf{R} \mapsto \mathbf{Z} \times \mathbf{R}$, there is $(\pi \circ t)(i) \geq t(i)$

Proof: The re-ordering operation works index after index in ascending order, starting from a point $i=0$. If $t(i) \geq t(i-$ 1 ), then the order between packet $i$ and packet $i-1$ is not changed by the transmission system, hence there is no need of re-ordering and $(\pi \circ t)(i)=t(i)$. But, if $t(i)<t(i-1)$, then a disorder occurs during the transmission, there is a need of re-ordering which maps $t(i)$ to a number no less than $t(i-$ $1)$, resulting in $(\pi \circ t)(i)>t(i)$.

QED.

How does the re-ordering affect the inter-arrival time and jitter? Using the knowledge obtained so far, we can draw the following conclusion for the inter-arrival time: For $x(i) \geq$ 0 , no re-ordering is needed, as there is no disorder. A reordering operation due when $x(i)<0$ is . Starting from $i$, one checks through $j=i, i+1, . ., i+m-1$ until $i+m$ with $x(i+$ $m) \geq 0$. Then, all those packets need to be buffered because they are out of order. Hence, the re-ordering operation is equivalent to setting $(\pi \circ x)(j):=0$ for $j \in[i, i+m-1]$ and $(\pi \circ x)(i+m):=x(i+m)-\sum_{j=i}^{i+m-1} x(j)$. Then, it follows

$\sum_{j=i}^{i+m}(\pi \circ x)(j)=x(i+m)-\sum_{j=i}^{i+m-1} x(j)$

Now, assume that the arrival after $i+m-1$ is ordered for $n>$ 0 consecutive packets, i.e. $x(j) \geq 0$ with $j \in[i+m, i+m+n]$ until again $x(i+m+n+1)<0$. Then

$\sum_{j=i+m}^{i+m+n}(\pi \circ x)(j)=\sum_{j=i+m}^{i+m+n} x(j)$

Thus, we obtain finally

$\sum_{j=i}^{i+m+n}(\pi \circ x)(j)=\sum_{j=i+m}^{i+m+n} x(j)-\sum_{j=i}^{i+m-1} x(j) \geq \sum_{j=i}^{i+m+n} x(j)$

meaning that the inter-arrival time tends to increase after the re-ordering operation at the receiver. The conclusion can be formalized as the following

Corollary $1 \lim _{n \rightarrow \infty} \operatorname{Pr}\left\{\frac{1}{n} \sum_{i=1}^{n}[(\pi \circ x)(i)-x(i)] \geq 0\right\}=1$

Proof:: Consider the set $N=\{1,2, \ldots, n\}$ of $n$ elements and $N=N^{+} \cup N^{-}$with $N^{+} \cap N^{-}=\emptyset$, where $N^{+}=\{i \in$ $N \mid x(i) \geq 0\}$ and $N^{-}=\{i \in N \mid x(i)<0\}$. Then

$\sum_{j \in N^{+}}[(\pi \circ x)(j)-x(j)]=0$

and

$\sum_{j \in N^{-}}[(\pi \circ x)(j)-x(j)]>-\sum_{j \in N^{-}} x(j) \geq 0$ where the equal sign applies when $N^{-}=\emptyset$. Hence

$E\left\{\frac{1}{n} \sum_{j \in N}[(\pi \circ x)(j)-x(j)]\right\} \geq 0$

Since the process is stationary, the relation holds for $n \rightarrow \infty$ and the weak convergence follows.

QED

\section{Compound transmission system}

A heterogeneous transmission path can be decomposed into mutual independent sections. We call such a transmission system a compound system, consisting of concatenated subsystems. In the chain of concatenated sub-systems the packet departure time at one sub-system is at the same time the packet arrival-time for the following sub-system. Thus, the delay observed at the output of the very last sub-system in the chain is the accumulation of delays from all sub-systems. Let $d_{k}(i)$ denote the packet delay caused by the $k$-th sub-system and $x_{k}(i)$ the corresponding inter-packet time. Denoting the random delay of the $i$-th packet of the compound system of $n$ sub-systems with $D_{n}(i)$, we have $D_{n}(i)=\sum_{k=1}^{n} d_{k}(i)$. Accordingly, the inter-packet time $X_{n}(i)$ of the $i$-th packet of the compound system can be determined by

$X_{n}(i)=D_{n}(i)-D_{n}(i-1)+\frac{1}{R_{n}}$

following the equation (1). Then,

$E\left\{X_{n}-\frac{1}{R_{n}}\right\}=\sum_{k=1}^{n} E\left\{x_{k}-\frac{1}{r_{k}}\right\}$

Therefore, we have the following result:

Proposition 3 For a compound system of $n$ independent concatenated sub-systems,

$E\left\{X_{n}\right\}=\frac{1}{R_{n}}$

when $E\left\{x_{k}\right\}=1 / r_{k}$ for $k=1,2, \ldots, n$. Then, the variances are subject to

$\operatorname{Var}\left\{X_{n}\right\}=\sum_{k=1}^{n} \operatorname{Var}\left\{x_{k}\right\}$.

The second statement follows from the independence between the sub-systems, i.e. $E\left\{\left(x_{k}-1 / r_{k}\right)\left(x_{i}-1 / r_{i}\right)\right\}=0$ for $k \neq i$, and it indicates a spreading of the variance as result of concatenation. Moreover, if $E\left\{d_{k}(i)-d_{k}(i-1)\right\}=0$ for all $i \in \mathbf{Z}$ and $k=1, . ., n$, we have $R_{n}=r_{k}=r$.

Corollary 2 If a compound system is the concatenation of $n$ independent sub-systems, then the compound system is rate preserving only when all $n$ sub-systems are rate preserving. 
At each inter-face between the sub-systems, the inter-packet time can be positive or negative. Negative inter-packet time means packet index disorder in the packet stream. The interpacket time after the re-ordering operation is always nonnegative.

\section{Data mining}

\subsection{Radio interfaces}

All modern wireless networks utilize the techniques of resource sharing over the air-interface. Users are multiplexed onto the frequency and time resources, using radio technologies such as CDMA (Code Division Multiple Access), TDMA (Time Division Multiple Access), OFDMA (Orthogonal Frequency Division Multiple Access) or any combination of them, to achieve spectrum efficiency and thus the network capacity. The radio-interface HSPA is based on CDMA with dynamic resource allocation. Similar to LTE, the protocol architecture of HSPA consists of, from bottom to top, PHY(Physical Layer) -MAC(Medium Access Control Protocol) - RLC(Radio Link Control Protocol) -PDCP(Packet Data Convergence Protocol) - IP(Internet Protocol), from bottom to top, with services carried in the payload of IP stack. A critical entity in the MAC layer is the HARQ that combines the request-response protocol and the adaptive channel coding to enhance the link performance. While improving the throughput of the radio channel, HARQ also introduces packet delay that varies with the changing radio channel condition. The merit is similar to that of a scheduler implemented at the radio network controller to assign radio resource among users and services, so that the air-interface can be fairly shared and the resource utilization be optimized. While scheduler and HARQ are essential for the performance of the radio interface, both introduce delay variation. Thus, through HARQ and the scheduler, the delay performance of a wireless network depends on both the radio channel condition and the cell traffic demand. As result, the jitter occurs. A project of 3GPP-RAN1 (2011) has provided ample simulation data for HSPA air interface performance in 3GPP-RAN1 (2006), in which traces of the packet delay and packet loss are recorded. Following 3GPP-SA4 (2006), the data were produced for the purpose of development of voice codec. As such, they are ideal for the study of the jitter behavior in an wireless cellular environment, because the underlying network configuration, channel conditions and traffic loads are predetermined and normalized by the standard development organizations, and as such are representative and authentic. We will make use of those data in the sequel.

\subsection{Stochastic sample paths}

In 3GPP-RAN1 (2006), an input stream of identical packets was sent every $20 \mathrm{~ms}$, and the packet delay and packet loss at the receiver were recorded for each packet. The samples
Table 2. Slope of the regression line as a two dimensional distribution.

\begin{tabular}{ccccc}
\hline $\mathrm{j}$ & $=$ & $\mathrm{ACF}$ & $\mathrm{PER}$ & $\mathrm{VAR}$ \\
$a(j, i)$ & $=$ & $-\alpha(\mathrm{ACF}, i)$ & $-\beta(\mathrm{PER}, i)$ & $-\gamma(\mathrm{VAR}, i)$ \\
\hline
\end{tabular}

for the down-link (DL) are collected on the RLC layer at the mobiles with a fixed geometry, while the samples for the up-link (UL) are collected on the RLC layer at RNC. The cellular network consists of 19 cells, each with 3 sectors. The 4 channel models used are based on ITU-R (2012):

$$
\begin{aligned}
& \text { - Pedestrian A with } 3 \mathrm{~km} \mathrm{~h}^{-1} \text { (PA3), } \\
& \text { - Pedestrian B with } 3 \mathrm{~km} \mathrm{~h}^{-1} \text { (PB3), } \\
& \text { - Vehicular A with } 30 \mathrm{~km} \mathrm{~h}^{-1} \text { (VA30), } \\
& \text { - Vehicular A with } 120 \mathrm{~km} \mathrm{~h}^{-1} \text { (VA120), }
\end{aligned}
$$

representing the different speed of mobility with respective channel model. In addition, 4 intensities of traffic load are considered:

- 40 users per sector (40U),

- 45 users per sector (45U),

- 60 users per sector (60U),

- 100 users per sector (100U).

The simulation was performed by two independent teams, using different simulators. As result, 54 traces were produced, among which 36 are for the down-link and 18 are for the up-link. Sequence of delay values in units of millisecond, together with the error flag are recorded in each trace, where the error flags indicate whether the packet is lost or in error. The latter is ignored because we assumed the proper error handling by the upper layer protocols. In order to make those data useful for our study, we have to transform these sequences into traces of inter-packet time after an re-ordering operation. The result of this preprocessing is referred to as sample path of inter-arrival time: A sample path is a sequence of real numbers representing the random inter-packet time, from which we can observe the jitter. Before evaluating the 54 sample paths, an introduction to fractional Brownian motion is due. The process of long range dependence has been extensively studied in the past and has found widely application Mandelbrot (1998); Beran (1994). Among the processes of long range dependence, the self-similar process is perhaps the mostly quoted in the information and telecommunication industry, e.g. by Paxson (1995); Geist (2000); Crovella et al. (1997); Taqqu et al. (1997); Willinger et al. (1997, 1994); Park et al. (2000). 
Table 3. Slope statistic for the DL $(\mathrm{df}=35)$.

\begin{tabular}{lrrr}
\hline Statistic & $a(\mathrm{ACF})$ & $a(\mathrm{PER})$ & $a(\mathrm{VAR})$ \\
\hline Mean & -0.39318 & -0.3601396 & -0.4713244 \\
Stdev & 0.223543 & 0.1697654 & 0.03629753 \\
$90 \%$-EM & 0.048695 & 0.036981 & 0.007907 \\
H & 0.803409 & 0.8199302 & 0.76433781 \\
\hline
\end{tabular}

Definition 2 A stochastic process $X(t)$ is self-similar when it has a distribution identical to that of $w^{-H} X(w t)$ for $t>0$, $w>0$ and $0.5<H<1$, where $H$ is called the Hurst parameter and $w$ the scaling factor. A stationary stochastic process is called a fractional Brownian motion when

1. $X(0)=0$

2. $X\left(t_{2}\right)-X\left(t_{1}\right)$ has a Gaussian distribution for $t_{1}<t_{2}$

3. $E\left\{X\left(t_{2}\right)-X\left(t_{1}\right)\right\}=0$

4. $\operatorname{Var}\left\{X\left(t_{2}\right)-X\left(t_{1}\right)\right\} \propto\left|t_{2}-t_{1}\right|^{H}$ for $1 / 2<H<1$

Based on this definition, long range dependence of stochastic process can be quantified by the Hurst parameter $H$, which can be estimated by means of proper asymptotes, as we will do in the following.

\subsection{Asymptotes}

For the packet inter-arrival time, the following second-order statistics can be computed using the sample paths:

1. Autocorrelation(ACF) :

$$
\rho(k)=\frac{\operatorname{Cov}\{x(\cdot+k) x(\cdot)\}}{\operatorname{Var}\{x(\cdot)\}}
$$

for $k \in \mathbf{Z}$ with $\rho(k)=O\left(k^{-\alpha}\right)$ for $k \rightarrow \infty$.

2. Periodogram(PER):

$$
p(\omega)=\frac{1}{2 \pi n}\left|\sum_{k=1}^{n}[x(k)-E\{x\}] e^{k \omega \sqrt{-1}}\right|^{2}
$$

for sufficiently large $n>1$ with $p(\omega)=O\left(\omega^{-\beta}\right)$ for $\omega \rightarrow 0$.

3. Variance (VAR):

$$
\sigma^{2}(k)=\operatorname{Var}\left\{\frac{1}{k} \sum_{i=1}^{k} x(i)\right\}
$$

for large $k>1$ with $\sigma^{2}(k)=O\left(k^{-\gamma}\right)$ for $k \rightarrow \infty$.
Table 4. Slope statistic for the UL ( $d f=17)$.

\begin{tabular}{lrrr}
\hline Statistic & $a(\mathrm{ACF})$ & $a(\mathrm{PER})$ & $a(\mathrm{VAR})$ \\
\hline Mean & -0.36799 & -0.2607483 & -0.4728221 \\
Stdev & 0.162307 & 0.2737584 & 0.02107574 \\
$90 \%$-EM & 0.050881 & 0.085819 & 0.006607 \\
$\mathrm{H}$ & 0.816006 & 0.6303742 & 0.76358897 \\
\hline
\end{tabular}

In the above asymptotes, the speed of the decay characterizes the dependence between entries in the sample path. A stochastic process is self-similar, when the sample estimate of $\rho(k)$ and $\sigma^{2}(k)$ tends to $k^{-\alpha}$ for large sample size $k$ and $\alpha>1 / 2$, or equivalently the sample estimate of $p(\omega)$ tends to $\omega^{-\beta}$ for $1<\beta<2$ for small $\omega$. The Hurst parameter $H$ used to characterize the self-similar process is related to the power of the asymptotes through $H=1+\alpha / 2=(1+\beta) / 2$. The stochastic process has the long range dependence when the corresponding Hurst parameter is in the range of $0.5<H<$ 1. In order to obtain an estimation of this parameter from our samples, we observe the slope of logarithm of the respective second order statistics, i.e. the parameter $a(\mathrm{ACR}, i)=\alpha$, $a(\mathrm{PER}, i)=\beta, a(\mathrm{VAR}, i)=\gamma$, respectively. In more general term, as shown in Table 2, we observe the two dimensional variables $a(j, i)$, where $j$ is the category index with $j=\mathrm{ACR}, \mathrm{PER}, \mathrm{VAR}$, and $i$ is the index of the sample path used.

Using all 54 samples, the intercept and the slope of the logarithm of the autocorrelation, the periodogram and the variance are estimated, respectively: All in total, 36 slopes are estimated for the down-link, while 18 slopes are estimated for the up-link. The sample mean of the slope $a(\mathrm{ACF}, i)$, $a(\mathrm{PER}, i)$ and $a(\mathrm{VAR}, i)$ are computed by averaging over $i$, resulting in

$a(\mathrm{ACF})=\frac{1}{n_{s}} \sum_{i=1}^{n_{s}} a(\mathrm{ACF}, i)$,

$a(\mathrm{PER})=\frac{1}{n_{s}} \sum_{i=1}^{n_{s}} a(\mathrm{PER}, i)$,

$a(\mathrm{VAR})=\frac{1}{n_{s}} \sum_{i=1}^{n_{s}} a(\mathrm{VAR}, i)$,

where $n_{s}$ is the number of samples. The results are shown in Tables 3 and 4 for the down-link and the up-link, respectively. The 90 percent confidence interval is also computed for Student- $t$ distribution and shown as the error margin, i.e. $90 \%$-EM in the tables.

As Tables 3 and 4 show, a long range dependence of the inter-packet time is visible. The Hurst parameter is estimated over the marginal distribution of three categories ACF, PER and VAR, and shown in Table 5. 
Table 5. Estimated $\mathrm{H}$.

\begin{tabular}{|c|c|c|c|}
\hline Link & $\begin{array}{r}\text { Hurst } \\
\text { Parameter }\end{array}$ & Stdev. & \#Samples \\
\hline DL & 0.796 & 5.26 & $86 \times 3=258$ \\
\hline UL & 0.747 & 1.20 & $18 \times 3=54$ \\
\hline
\end{tabular}

\section{Mobile-to-mobile connection}

A mobile-to-mobile connection requires at least an up-link, a down-link and at least an intermediate node representing the core network. However, since we only have sample paths for the radio interface, it is reasonable to focus our attention to the jitters caused by the air-interface on the jitter. For this purpose, the model can be simplified by assuming that the core network processes the incoming traffic according to a deterministic round-robin scheduler. As result, the observed statistics of the mobile traffic will not be affected by the other traffics that join the node in the core network, which adds only a fixed delay to the packet stream of the refrence mobile traffic. Thus, the inter-arrival time, hence the jitter, becomes independent of the core network under this assumption, and we are left with a concatenation of only two sub-systems: One is the up-link radio interface and the other is the down-link radio interface. Using data available in 3GPP-RAN1 (2006) for up-link and down-link, one can construct new sample paths to study the jitter statistics of an end-to-end connection. Now that the compound system consists of two concatenated sub-systems, 4 groups of new sample paths can be generated, by combining four different types of sub-systems, i.e. low mobility ( $\mathrm{lm})$, high mobility (hm), low traffic (lt), high traffic (ht). The existing sample paths can, according to their respective physical configuration, be associated with these 4 groups as the following,

1. Im-lt: low mobility and low traffic (PA3,PB3,VA30; 40U)

2. hm-lt: high mobility and low traffic (VA120; 40U)

3. lm-ht: low mobility and high traffic (PA3, PB3,VA30; 100U)

4. hm-ht: high mobility and high traffic (VA120; 100U)

for the down-link and as

1. up-lm: low mobility (PA3,PB3,VA30)

2. up-hm: high mobility(VA120)

for the up-link. Since the inter-packet time of the subsystems demonstrates long range dependence, we are interested in the same long range dependence of the inter-packet time in a compound system. To find more about this, let $\rho(k)$ denote the autocorrelation function of the inter-packet
Table 6. Estimated $\alpha_{c}$ of the mobile-to-mobile connections.

\begin{tabular}{ccc}
\hline Mean & Stdev & $90 \%$ EM \\
\hline 0.078454 & 0.369239 & 0.027325
\end{tabular}

time of the compound system, and $\rho_{i}(k)$ be the autocorrelation function of the inter-packet time of the sub-systems with $i=1,2, \ldots m$. When independence among the sub-systems is assumed, we have

$\rho(k)=\sum_{i=1}^{m} \rho_{i}(k) w_{i}$

for any $k \geq 0$, where

$w_{i}=\frac{\operatorname{Var}\left(x_{i}\right)}{\sum_{i=1}^{m} \operatorname{Var}\left(x_{i}\right)}$

By arranging the terms on the right hand in ascending order of magnitude $\alpha_{i}$

$$
\begin{aligned}
\lim _{k \rightarrow \infty} \rho(k)= & \sum_{i=1}^{m} \lim _{k \rightarrow \infty} \rho_{i}(k) w_{i}=k^{-\alpha_{c}}\left(w_{1} c_{1}+\right. \\
& \left.w_{2} c_{2} k^{-\alpha_{2}+\alpha_{c}}+\ldots+w_{m} c_{m} k^{-\alpha_{m}+\alpha_{c}}\right)
\end{aligned}
$$

for $\alpha_{c}=\min _{1 \leq i \leq m} \alpha_{i}$ and $c_{i}>0$ with $i=1,2, . . m$. This indicates that the asymptote of the auto-correlation is dominated by the sub-system with the slowest decay. As an example we evaluate the parameter of the concatenated system consisting of 18 up-link samples and 18 down-link samples, randomly selected from the available data base. Combining them to make an end-to-end connection results in $18 \times 18=324 \mathrm{sam}$ ples. Now parameter $\alpha_{c}$ is estimated using these $324 \mathrm{sam}-$ ples, the result is shown in Table 6. Comparing to the numbers in Table 3 and 4, the estimated $\alpha_{c}$ in Table 6 is less than both $\alpha_{0}$ and $\alpha_{1}$. Thus, the example is consistent with the analysis. On the other hand, when the constants $c_{i}$ are also taken into account, we obtain the following more accurate result:

Proposition 4 For a compound system of $m$ concatenated independent sub-systems, assume the processes of interdeparture time $x_{i}$ are pair-wise independent for $i=1,2, . ., m$, and the autocorrelation function $\rho_{i}(k)$ for the $i$-th subsystem has the asymptotic $\lim _{k \rightarrow \infty} \rho_{i}(k)=c_{i} k^{-\alpha_{i}}$ with $c_{i}>0$ and $\alpha_{i}>0$. Then, the autocorrelation function of the compound system $\rho(k)$ has the asymptotic behavior

$\lim _{k \rightarrow \infty} \rho(k)=O\left(k^{-\alpha_{c}}\right)$

with

$\alpha_{c} \leq \frac{\sum_{i=1}^{m} \alpha_{i} c_{i} w_{i}}{\sum_{i=1}^{m} c_{i} w_{i}}$. 
Proof: Introducing $c_{w}=\sum_{i=1}^{m} c_{i} w_{i}$ in (18) we obtain

$\lim _{k \rightarrow \infty} \rho(k)=c_{w} \sum_{i=1}^{m} k^{-\alpha_{i}} c_{i} w_{i} / c_{w}$

The sum on the right hand of the equation can be viewed as the expectation of the convex function $k^{-u}$. By Jensen's inequality,

$k^{-\min _{i \leq i \leq m}\left(\alpha_{i}\right)} \geq \sum_{i=1}^{m} k^{-\alpha_{i}} \frac{c_{i} w_{i}}{c_{w}} \geq k^{-\sum_{i=1}^{m} \alpha_{i} \frac{c_{i} w_{i}}{c_{w}}}$

for $c_{w}>0$ and $\alpha_{i}>0$. Since $\ln \left(k^{-u}\right) \geq \ln \left(k^{-v}\right)$ for $u \leq v$,

$\lim _{k \rightarrow \infty} \frac{-\ln [\rho(k)]}{\ln [k]} \leq \sum_{i=1}^{m} \alpha_{i} \frac{c_{i} w_{i}}{c_{w}}$

Obviously, the term on the left hand is $\alpha_{c}$ and the equal sign holds when $\alpha_{c}=\alpha_{i}$ for $i=1,2, \ldots, m$.

QED.

\section{Conclusions}

So far we have studied the jitter statistics experienced by the real time service like voice, when packet streams are transmitted over HSPA wireless systems, using analytical as well as empirical methods. By analysis we established the relationship between delay and inter-packet time, and investigated the stochastic behavior of those quantities. With help of simulation data available for HSPA, we could estimate the second order statistics of the jitter and evaluate their asymptotes. Combining the existing samples to construct new samples representing new physical scenarios allows us further to investigate the jitter statistics of a mobile-to-mobile connection.

An important assumption made here was that the service provided by the packet stream requires rate preservation, i.e. the data are generated with constant rate and also expected to be received with the same rate. This requirement makes it necessary to deploy buffer to enable re-ordering at the receiving end. Only the operation of re-ordering can mitigate the delay jitter introduced by the deployment of the scheduler and the HARQ in a wireless transmission system. Evaluation of the sample paths with respect to the asymptotic decay of the autocorrelation, the periodogram and the variance reveals a statistical long range dependence of the inter-packet time, where the power law of the asymptote of the order $k^{-\alpha}$ has typically $1 / 2<\alpha<1$. For a compound system consisting of concatenated sub-systems, the long range dependence behavior of the compound system is dominated by the sub-system with the slowest decay, a finding confirmed by the sample evaluation.

\section{References}

3GPP(Third Generation Partnership Project): “TS 36.300 V8.8.0”, available at: http://www.3gpp.org, Aug. 2012.

3GPP: "LS on RAB and the error-delay-profile for the performance characterization of VoIMS over HSDPA/EULLS", RAN1-44, 13-17 Febuary 2006, Denver, avilable at: http://www.quintillion.co.jp/3GPP/TSG_RAN/ TSG_RAN2006/TSG_RAN_WG1_RL1_2.html, Aug. 2012.

3GPP, "Specification 25 Series", RAN1, available at: http://www. 3gpp.org July, 2011.

3GPP: "Working Document for the Performance Characterization of VoIMS over HSDPA/EDCH, v0.0.4", 3GPP-SA4-40, 28 August-2 September 2006, Sophia-Antipolis, available at: http://www.3gpp.org, Dec. 2006.

International Telecommunications Union, "Guidelines for Evaluation of Radio Transmission”, Recommendation ITU-R M.1225, available at: http://www.itu.int/rec/R-REC-M.1225/en, April, 2012.

Paxson, V.: "Fast Approximation of Self-Similar Network Traffic", LBL-36750, Lawrence Berkeley Laboratory, April, 1995.

Geist, R. and Westall, J.: "Practical Aspects of Simulating Systems Having Arrival Processes with Long-range Dependence", IEEE Proc. of the 2000 Winter Simulation Conference, 2000.

Mandelbrot, B. B.: Mutifractals and 1/f Noise, Springer-Verlag, 1998.

Beran, J.: Statistics for Long-Memory Processes, Chapman and Hall, 1994.

Crovella, M. E. and Bestavros, A.: Self-Similarity in World Wide Web Traffic: Evidence and Possible Causes, IEEE/ACM, Trans. on Networking, 5(6), December, 1997

Taqqu, M., Willinger, W., and Sherman, R.: Proof of a Fundamental Results in Self-Similar Traffic Modeling, ACM SiGCOMM Compiuter Communication Review, 27(2), 5-23, April, 1997.

Willinger, W., Taqqu, M. S., Sherman, R., and Wilson, D. V.: SelfSimilar Through High-Variability: Statistical Analysis of Ethernet LAN Traffic at the Source Level, IEEE/ACM, Trans. on Networking, 5(1), February, 1997.

Willinger, W., Taqqu, M. S., Sherman, R., and Wilson, D. V.: On the Self-Similar Nature of Ethernet Traffic (Extended Version), IEEE/ACM Trans. on Networking. 2(1), 1-15, February, 1994.

Park, K. and Willinger, W. (Ed.): Self-Similar Network Traffic and Performance Evaluation, John Wiley and Sons, Inc., 2000. 